\title{
The effect of immunogenetic factors on productive longevity and reproductive qualities of cows
}

\author{
Lyubov Efimova*, Elena Gatilova, Evgeny Ivanov, and Vera Tereshchenko
}

Krasnoyarsk Research Institute for Animal Husbandry - Separate Division of the Federal State Budget Scientific Institution "Federal Research Center "Krasnoyarsk Science Center of the Siberian Branch of the Russian Academy of Sciences", Krasnoyarsk, 660049, Russia

\begin{abstract}
Increasing the productive longevity of cows with high reproductive ability is an urgent task in dairy cattle breeding both in Russia and foreign countries. Research was conducted in Krasnoyarsk region. The object was retired cows $(n=484)$. The blood groups of cows, indicators of productive longevity, and reproductive ability of cows were studied. The number of antigen carriers and non-carriers (frequency $30.0 \%$ and more) was analyzed. Out of 21 antigens with a frequency of more than $30.0 \%$, the most frequently encountered antigens were $\mathrm{E}(80.6 \%), \mathrm{H}^{`}(77.5 \%), \mathrm{L}(58.5 \%), \mathrm{W}(57.6 \%), \mathrm{A}_{2}(57.0 \%), \mathrm{Z}$ $(54.1 \%), \mathrm{C}(52.5 \%)$ and $\mathrm{X}_{2}(50.4 \%)$. The cows carrying $\mathrm{O} 1$ antigen had the highest productive longevity, the difference from the cows not carrying this antigen by the retirement and lactation age was 0.8 years (p $<0.001$ ), and the period of economic use was 9.5 months ( $p<0.001$ ), life-long milk yield was $3852 \mathrm{~kg}$ (p $<0.001$ ). Cows carrying antigens $\mathrm{G}_{3}, \mathrm{O}_{1}$, and $\mathrm{L}^{\prime}$ had the best reproductive ability. In carriers of antigens $\mathrm{O}_{1}$, $\mathrm{G}_{3}$, and $\mathrm{E}_{3}$, the service period was lower by $22.9 ; 20.3$ and 18.9 days ( $<<0.05$ ), and the fertility index was higher by $1.8 ; 1.6$ and 1.7 units ( $\mathrm{p}<0.05)$. In cows carrying $\mathrm{L}^{\prime}$ antigen, the service period was shorter by 16.9 days $(\mathrm{p}<0.05)$
\end{abstract}

\section{Introduction}

For the cost-effective dairy cattle breeding and high milk productivity, it is necessary to use feed additives in the diet of lactating cows, including those containing local raw materials. It is necessary to reproduce tribal mother cows and father bulls. Implementation of the genetic potential of milk productivity [1] is impossible without various biologically active additives which increase productivity and normalize indicators of homeostasis.

An important task of the dairy cattle breeding is an increase in the economic use of cows [2] while maintaining their high productivity and fecundity associated with economic benefits that directly depend on these indicators. Over the years, both Russian and foreign scientists have shown interest in this issue.

In 2013, T. Pritchard et al. [3] argued that direct selection of cows reduces mastitis and lameness and improves a reproductive ability and increases the life expectancy. The more fertile the cow, the shorter the service period, the lesser number of inseminations is needed for one fruitful one [4]. V. Deeva says about the influence of the first calving age on the life expectancy of [5]. Russian and Ukrainian researchers have found that productive longevity depends on genetic factors such as linear affiliation [6], blood count [7], and maternal milk production [8]. According to G. Serdyuk, many researchers agree that the optimal blood count should not exceed $75 \%$ [7].
V.I. Shlyakhtunov and E.M. Karpovich [9] found that father bulls are of great importance for these indicators. The authors believe that the contribution of father bulls to the genetic improvement of dairy herds is $75-85 \%$. Other researchers [10] report that most researchers and breeders rate the impact of bulls as $90 \%$. M.A. Pilipenko [11] notes that the productive longevity of cows depends on the individual characteristics of fathers (up to 70\%). In addition, according to the author, the productive longevity of the bulls can have a significant impact on these indicators.

O.R. Kurechenkova et al. [12] analyzed the productive longevity of cows of the Red Steppe breed (Siberian and Kulundinsky types), who are daughters of bulls born in different countries: Russia, Germany, Denmark, and Canada. They found that daughters of the bulls born in Russia had the longest age of productive longevity (4.74 lactations), which exceeded one of their peers by $0.57-1.21$ lactations. The authors explain the results by the fact that the daughters of the bulls born in Russia are most adapted to the feeding and keeping conditions, and show the best results.

A large number of studies are devoted to the search for erythrocyte and molecular genetic markers of economically valuable traits that can improve efficiency and accelerate the selection process. Russian and foreign scientists suggested using molecular genetic markers of productive longevity and reproductive ability of cows of different breeds [13-15].

Corresponding author: krasniptig75@ yandex.ru 
N.S. Yudin et al. developed a DNA panel for selecting SNPs during the genetic analysis of economically useful traits; it can be used to estimate frequencies of alleles in populations [16].

G.P. Kovaleva et al. [17] found that cows of BlackMotley breed with different antigens in the B blood system genotype differed by the duration of productive use and lifelong productivity. The difference between the cows with the maximum productive life and the cows having the minimum values amounted to 0.81 lactations, by the life-long milk yield $-7039.8 \mathrm{~kg}$, by the amount of milk fat $-269.2 \mathrm{~kg}$.

O.S. Shatalina [18] established the relationship between the antigenic similarity of parental pairs and the inheritance of blood antigens by descendants with their reproductive characteristics. The research object was holsteinized Black-Motley Ural cows.

Many researchers have studied the genetic structure of cows of different breeds by the blood groups, the number of genetic systems, alleles, and genotypes at each locus $[19,20]$, as well as the relationship of erythrocyte antigens [21, 22] and blood group alleles with milk production and animal reproductive ability [23]. A lot of antigen markers of high and low indices were isolated.

Russian researchers have been identifying marker antigens with high productive longevity. This concerns Black-Motley [24], Aishir [25], and other breeds common in the European part of Russia. For cows of Red-Motley breed, this aspect remains unstudied.

The article aims to identify antigen markers of productive longevity and good reproductive ability of cows of Red-Motley breed.

\section{Material and methods}

The studies were conducted at SC "Arefevskoye" in Krasnoyarsk region. The material for the study was data on retired cows $(n=484)$ for the period from 2013 to 2018. The data were obtained using the SELEX program. Studies on the determination of blood groups of cows were conducted in immunogenetic examination laboratory BC "Krasnoyarskagroplem". For serological tests, standard reagents determining 55 antigenic factors in 11 systems (loci) of blood groups were used.

Blood groups and antigen frequency were studied, indicators of productive longevity (age of retirement in years and lactations, a period of economic use (PEU) in months, life-time milk yield), and reproductive ability of cows (the first calving age, the service period and the fertility index) were determined. The compared groups were carriers and non-carriers of antigens.

The frequency of occurrence of antigens (p) is calculated by formula:

$$
p=F / n \cdot 100,
$$

where $\mathrm{p}$ - frequency of occurrence of antigens, \%; F number of cows having an antigen; $\mathrm{n}$ - total number of animals.

The fertility index (PI) is determined by the Doha's formula:

$$
P I=100-(F C A+2 I P),
$$

where FCA - first calving age (months), IP intercalving period (months). All the data were processed using the methods of variation statistics. The significance of the difference between the groups was determined by the t-Student's test.

\section{Results and discussion}

\subsection{The frequency of occurrence of antigens in the herd}

58 antigens were identified, 21 antigens occurred with the highest frequency (more than 30.0\%). The classification of cows into carriers and non-carriers of 21 antigens is presented in Table 1.

Table 1. The classification of cows into antigen carriers and

\begin{tabular}{|c|c|c|}
\hline $\begin{array}{c}\text { Antigen } \\
(+/-)\end{array}$ & $\mathrm{n}$ & Frequency, \% \\
\hline $\mathrm{A}_{1}(+)$ & 162 & 33.5 \\
\hline $\mathrm{A}_{1}(-)$ & 322 & 66.5 \\
\hline $\mathrm{A}_{2}(+)$ & 276 & 57.0 \\
\hline $\mathrm{A}_{2}(-)$ & 208 & 43.0 \\
\hline $\mathrm{G}_{3}(+)$ & 166 & 34.3 \\
\hline $\mathrm{G}_{3}(-)$ & 318 & 65.7 \\
\hline $\mathrm{O}_{1}(+)$ & 149 & 30.8 \\
\hline $\mathrm{O}_{1}(-)$ & 335 & 69.2 \\
\hline $\mathrm{Q}(+)$ & 194 & 40.1 \\
\hline $\mathrm{Q}(-)$ & 290 & 59.9 \\
\hline $\mathrm{Y}_{2}(+)$ & 168 & 34.7 \\
\hline$Y_{2}(-)$ & 316 & 65.3 \\
\hline $\mathrm{A}_{2}{ }_{2}(+)$ & 207 & 42.8 \\
\hline $\mathrm{A}_{2}{ }_{2}(-)$ & 277 & 57.2 \\
\hline $\mathrm{E}_{3}^{\prime}{ }_{3}(+)$ & 191 & 39.5 \\
\hline$E_{3}{ }_{3}(-)$ & 293 & 60.5 \\
\hline $\mathrm{C}_{1}(+)$ & 146 & 30.2 \\
\hline $\mathrm{C}_{1}(-)$ & 338 & 69.8 \\
\hline $\mathrm{C}_{2}(+)$ & 254 & 52.5 \\
\hline $\mathrm{C}_{2}(-)$ & 230 & 47.5 \\
\hline $\mathrm{E}(+)$ & 390 & 80.6 \\
\hline E (-) & 94 & 19.4 \\
\hline$X_{1}(+)$ & 154 & 31.8 \\
\hline$X_{1}(-)$ & 330 & 68.2 \\
\hline $\mathrm{X}_{2}(+)$ & 244 & 50.4 \\
\hline$X_{2}(-)$ & 240 & 49.6 \\
\hline $\mathrm{R} 2(+)$ & 179 & 37.0 \\
\hline $\mathrm{R}_{2}(-)$ & 305 & 63.0 \\
\hline $\mathrm{W}(+)$ & 279 & 57.6 \\
\hline W (-) & 205 & 42.4 \\
\hline$L^{\prime}(+)$ & 176 & 36.4 \\
\hline$L^{\prime}(-)$ & 308 & 63.6 \\
\hline $\mathrm{J}(+)$ & 210 & 43.4 \\
\hline $\mathrm{J}(-)$ & 274 & 56.6 \\
\hline $\mathrm{L}(+)$ & 283 & 58.5 \\
\hline $\mathrm{L}(-)$ & 201 & 41.5 \\
\hline $\mathrm{S}_{2}(+)$ & 148 & 30.6 \\
\hline $\mathrm{S}_{2}(-)$ & 336 & 69.4 \\
\hline $\mathrm{H}^{\prime}(+)$ & 375 & 77.5 \\
\hline $\mathrm{H}^{\prime}(-)$ & 109 & 22.5 \\
\hline
\end{tabular}
non-carriers (+/-). 


\begin{tabular}{|c|c|c|}
\hline $\mathrm{Z}(+)$ & 262 & 54.1 \\
\hline $\mathrm{Z}(-)$ & 222 & 45.9 \\
\hline
\end{tabular}

The following antigens were found with a frequency of more than 50\%: E (80.6\%), H $(77.5 \%), \mathrm{L}(58.5 \%), \mathrm{W}$ (57.6\%), $\mathrm{A}_{2}(57.0 \%), \mathrm{Z}(54.1 \%), \mathrm{C}(52.5 \%)$ and $\mathrm{X}_{2}$ $(50.4 \%)$. Our data are comparable with the results obtained by N. Ya. Kulumaeva and G.M. Goncharenko [26] for Simmental cows, in particular with the results on antigens $\mathrm{E}, \mathrm{H}, \mathrm{W}, \mathrm{A}_{2}$, and $\mathrm{Z}$ (the frequency of occurrence varied from 59.0 to $97.0 \%$ ).

\subsection{Productive longevity depending on the presence of the antigen}

The cows were excluded from the herd due to their barrenness (198 cows or 40.9\%), low productivity (104 cows or $21.5 \%$ ), udder diseases (68 cows or $14.0 \%$ ) and difficulties of births (36 cows or $7.4 \%$ ). There was no dependence of exclusion causes of the presence of any antigens.

Productive longevity indicators for carriers and noncarriers of antigens are shown in Table 2.

The cows carrying $\mathrm{O} 1$ antigen had the highest productive longevity, their productive longevity was higher than that of the non-carriers by 0.8 years ( $p$ $<0.001$ ), and the period of economic use was larger by 9.5 months ( $\mathrm{p}<0.001$ ), the life-long milk yield was larger by $3852 \mathrm{~kg}(\mathrm{p}<0.001)$. Non-carriers of antigens $A_{1}, A_{2}, G_{3}$ and $X_{1}$ were superior to the carriers by the retirement and lactation age by $0.4-0.7$ years $(p<0.05$ 0.01 ), by the period of economic use - by 5.9-7.4 months ( $\mathrm{p}<0.01$ ), by the life-long milk yield - by $2480-2765 \mathrm{~kg}$ ( $\mathrm{p}<0.05-0.01)$.

\subsection{Reproductive ability depending on the presence of the antigen}

The best reproductive ability was observed in the carriers of antigens $\mathrm{G}_{3}, \mathrm{O}_{1}$, and $\mathrm{L}^{\prime}$. In carriers of $\mathrm{G}_{3}$ antigen, the service period was shorter by 20.3 days, and the fertility index was higher by $1.6(\mathrm{p}<0.05)$. In carriers of antigens $\mathrm{O}_{1}$ and $\mathrm{E}_{3}^{\prime}$, the service period was shorter by 22.9 and 18.9 days, respectively, and the fertility index was 1.8 and 1.7 more $(p<0.05)$. In carriers of $L^{\prime}$ antigen, the service period was shorter by 16.9 days $(\mathrm{p}<0.05)$ (Table $3)$.

By the early maturity indicator (the minimum age of first calving), the best indicators were observed in the carriers of $\mathrm{X}_{1}$ antigen $(-1.3$ months; $\mathrm{p}<0.01)$ and noncarriers of $\mathrm{C}_{2}$ antigen $(-1$ month; $\mathrm{p}<0.05)$.

S.L. Gridina et al. [27] found that the reproductive ability of cows is better in the absence of $J$ antigen. In our studies, the difference in the reproductive ability between carriers and non-carriers of $\mathbf{J}$ antigen was statistically insignificant.
Table 2. Productive longevity of cows depending on the antigen carrier state.

\begin{tabular}{|c|c|c|c|c|}
\hline \multirow{2}{*}{\begin{tabular}{|c|} 
Antige \\
$\mathrm{n}$ \\
$(+/-)$ \\
\end{tabular}} & \multicolumn{2}{|c|}{ Age } & \multirow{2}{*}{$\begin{array}{l}\text { PHI *, } \\
\text { months }\end{array}$} & \multirow{2}{*}{$\begin{array}{c}\text { Lifetime milk } \\
\text { yield, } \mathrm{kg}\end{array}$} \\
\hline & years & lactations & & \\
\hline$A_{1}(+)$ & \begin{tabular}{|c|}
$5.4 \pm 0.1$ \\
5
\end{tabular} & $\begin{array}{c}3.0 \pm 0.1 \\
4\end{array}$ & $\begin{array}{c}41.2 \pm 1.8 \\
2\end{array}$ & $\begin{array}{c}18472 \pm 852 \\
.8\end{array}$ \\
\hline$A_{1}(-)$ & \begin{tabular}{|c|}
$6.1 \pm 0.1$ \\
$2^{* *}$
\end{tabular} & $\begin{array}{r}3.7 \pm 0.1 \\
1^{* * *}\end{array}$ & $\begin{array}{c}48.6 \pm 1.3 \\
4^{* *}\end{array}$ & $\begin{array}{c}21237 \pm 611 \\
.3^{* *}\end{array}$ \\
\hline $2(+)$ & \begin{tabular}{|c|}
$5.7 \pm 0.1$ \\
2 \\
\end{tabular} & $\begin{array}{c}3.3 \pm 0.1 \\
1\end{array}$ & $\begin{array}{c}43.6 \pm 1.4 \\
0\end{array}$ & $\begin{array}{c}19246 \pm 657 \\
.0\end{array}$ \\
\hline $\mathrm{A}_{2}(-)$ & \begin{tabular}{|c|}
$6.1 \pm 0.1$ \\
$5^{*}$ \\
\end{tabular} & \begin{tabular}{|c|}
$3.7 \pm 0.1$ \\
$4^{*}$ \\
\end{tabular} & $\begin{array}{c}49.5 \pm 1.7 \\
0^{* *} \\
\end{array}$ & $\begin{array}{c}21726 \pm 760 \\
.9^{*} \\
\end{array}$ \\
\hline $\mathrm{G}_{3}(+)$ & \begin{tabular}{|c|}
$5.5 \pm 0.1$ \\
5
\end{tabular} & \begin{tabular}{|c|}
$3.1 \pm 0.1$ \\
4
\end{tabular} & $\begin{array}{c}41.6 \pm 1.7 \\
8\end{array}$ & $\begin{array}{c}18522 \pm 847 \\
.1\end{array}$ \\
\hline $\mathrm{G}_{3}(-)$ & \begin{tabular}{|c|}
$6.1 \pm 0.1$ \\
$2^{* *}$ \\
\end{tabular} & \begin{tabular}{|c|}
$3.6 \pm 0.1$ \\
$1^{* *}$ \\
\end{tabular} & $\begin{array}{c}48.5 \pm 1.3 \\
6^{* *}\end{array}$ & $\begin{array}{c}21246 \pm 613 \\
.6^{* *} \\
\end{array}$ \\
\hline$D_{1}(+)$ & \begin{tabular}{|c|}
$6.4 \pm 0.1$ \\
$7^{* * *}$ \\
\end{tabular} & $\begin{array}{r}4.0 \pm 0.1 \\
6^{* * *} \\
\end{array}$ & $\begin{array}{c}52.7 \pm 2.0 \\
0^{* * *}\end{array}$ & $22978 \pm 912$ \\
\hline $\mathrm{O}_{1}(-)$ & \begin{tabular}{|c|}
$5.6 \pm 0.1$ \\
1
\end{tabular} & \begin{tabular}{|c|}
$3.2 \pm 0.1$ \\
0
\end{tabular} & $\begin{array}{c}43.2 \pm 1.2 \\
7\end{array}$ & $\begin{array}{c}19126 \pm 587 \\
.2\end{array}$ \\
\hline $\mathrm{Q}(+)$ & \begin{tabular}{|c|}
$5.8 \pm 0.1$ \\
5
\end{tabular} & \begin{tabular}{|c|}
$3.4 \pm 0.1$ \\
4
\end{tabular} & $\begin{array}{c}45.4 \pm 1.7 \\
4\end{array}$ & $\begin{array}{c}20081 \pm 807 \\
.4\end{array}$ \\
\hline $\mathrm{Q}(-)$ & \begin{tabular}{|c|}
$5.9 \pm 0.1$ \\
2 \\
\end{tabular} & \begin{tabular}{|c|}
$3.5 \pm 0.1$ \\
1
\end{tabular} & $\begin{array}{c}46.6 \pm 1.4 \\
0\end{array}$ & $\begin{array}{c}20466 \pm 636 \\
.8 \\
\end{array}$ \\
\hline$Y_{2}(+)$ & \begin{tabular}{|c|}
$5.8 \pm 0.1$ \\
5 \\
\end{tabular} & \begin{tabular}{|c|}
$3.4 \pm 0.1$ \\
4 \\
\end{tabular} & $\begin{array}{c}45.6 \pm 1.7 \\
8 \\
\end{array}$ & $\begin{array}{c}20291 \pm 818 \\
.7 \\
\end{array}$ \\
\hline$Y_{2}(-)$ & \begin{tabular}{|c|}
$5.9 \pm 0.1$ \\
2 \\
\end{tabular} & \begin{tabular}{|c|}
$3.5 \pm 0.1$ \\
1 \\
\end{tabular} & $\begin{array}{c}46.4 \pm 1.3 \\
8 \\
\end{array}$ & $\begin{array}{c}20323 \pm 630 \\
.8 \\
\end{array}$ \\
\hline $\mathrm{A}_{2}(+)$ & \begin{tabular}{|c|}
$5.7 \pm 0.1$ \\
4 \\
\end{tabular} & \begin{tabular}{|c|}
$3.2 \pm 0.1$ \\
3 \\
\end{tabular} & $\begin{array}{c}43.8 \pm 1.6 \\
2 \\
\end{array}$ & $\begin{array}{c}19325 \pm 724 \\
.5 \\
\end{array}$ \\
\hline $2 t^{-1}$ & \begin{tabular}{|c|}
$6.0 \pm 0.1$ \\
3 \\
\end{tabular} & \begin{tabular}{|c|}
$3.6 \pm 0.1$ \\
$2^{*}$
\end{tabular} & $\begin{array}{c}47.9 \pm 1.4 \\
7 \\
\end{array}$ & $\begin{array}{c}21049 \pm 683 \\
.2\end{array}$ \\
\hline $\mathrm{E}_{3}{ }_{3}(+)$ & \begin{tabular}{|c|}
$5.8 \pm 0.1$ \\
4
\end{tabular} & \begin{tabular}{|c|}
$3.4 \pm 0.1$ \\
2
\end{tabular} & $\begin{array}{c}45.8 \pm 1.5 \\
6\end{array}$ & $\begin{array}{c}20536 \pm 727 \\
.6\end{array}$ \\
\hline $3(-)$ & \begin{tabular}{|c|}
$5.9 \pm 0.1$ \\
3 \\
\end{tabular} & \begin{tabular}{|c|}
$3.5 \pm 0.1$ \\
2 \\
\end{tabular} & $\begin{array}{c}46.3 \pm 1.4 \\
9 \\
\end{array}$ & $\begin{array}{c}20165 \pm 676 \\
.8 \\
\end{array}$ \\
\hline $\mathrm{C}_{1}(+)$ & \begin{tabular}{|c|}
$5.8 \pm 0.1$ \\
6 \\
\end{tabular} & $\begin{array}{c}3.3 \pm 0.1 \\
5\end{array}$ & $\begin{array}{c}44.6 \pm 1.8 \\
9\end{array}$ & $\begin{array}{c}19651 \pm 899 \\
.4\end{array}$ \\
\hline $\mathrm{C}_{1}(-)$ & $\begin{array}{c}5.9 \pm 0.1 \\
2\end{array}$ & \begin{tabular}{|c|}
$3.5 \pm 0.1$ \\
1
\end{tabular} & $\begin{array}{c}46.8 \pm 1.3 \\
3 \\
\end{array}$ & $\begin{array}{c}20597 \pm 601 \\
.4\end{array}$ \\
\hline $\mathrm{C}_{2}(+)$ & \begin{tabular}{|c|}
$5.9 \pm 0.1$ \\
3 \\
\end{tabular} & \begin{tabular}{|c|}
$3.4 \pm 0.1$ \\
3 \\
\end{tabular} & $\begin{array}{c}45.4 \pm 1.5 \\
6 \\
\end{array}$ & $\begin{array}{c}20006 \pm 718 \\
.0 \\
\end{array}$ \\
\hline $\mathrm{C}_{2}(-)$ & \begin{tabular}{|c|}
$5.9 \pm 0.1$ \\
3 \\
\end{tabular} & \begin{tabular}{|c|}
$3.5 \pm 0.1$ \\
2 \\
\end{tabular} & $\begin{array}{c}46.9 \pm 1.5 \\
2 \\
\end{array}$ & $\begin{array}{c}20649 \pm 692 \\
.2 \\
\end{array}$ \\
\hline$E(+)$ & \begin{tabular}{|c|}
$5.8 \pm 0.1$ \\
0 \\
\end{tabular} & \begin{tabular}{|c|}
$3.4 \pm 0.0$ \\
9 \\
\end{tabular} & $\begin{array}{c}45.4 \pm 1.1 \\
8 \\
\end{array}$ & $\begin{array}{c}20082 \pm 538 \\
.9 \\
\end{array}$ \\
\hline $\mathrm{E}(-)$ & \begin{tabular}{|c|}
$6.2 \pm 0.2$ \\
4 \\
\end{tabular} & \begin{tabular}{|c|}
$3.7 \pm 0.2$ \\
2 \\
\end{tabular} & $\begin{array}{c}49.2 \pm 2.7 \\
7 \\
\end{array}$ & $\begin{array}{c}21264 \pm 127 \\
6.6 \\
\end{array}$ \\
\hline $1+$ & $\begin{array}{c}5.3 \pm 0.1 \\
5\end{array}$ & $\begin{array}{c}3.0 \pm 0.1 \\
3\end{array}$ & $\begin{array}{c}40.4 \pm 1.6 \\
6\end{array}$ & $\begin{array}{c}18260 \pm 816 \\
.4\end{array}$ \\
\hline
\end{tabular}




\begin{tabular}{|c|c|c|c|c|}
\hline$X_{1}(-)$ & $\begin{array}{l}.1 \pm 0.1 \\
2^{* * *}\end{array}$ & $\begin{array}{r}3.7 \pm 0.1 \\
1^{* * *}\end{array}$ & $\begin{array}{c}48.8 \pm 1.3 \\
8^{* * *}\end{array}$ & $21269 \pm 620$ \\
\hline$X_{2}(+)$ & $\begin{array}{c}5.8 \pm 0.1 \\
3\end{array}$ & $\begin{array}{c}3.4 \pm 0.1 \\
2\end{array}$ & $\begin{array}{c}45.4 \pm 1.5 \\
5\end{array}$ & $\begin{array}{c}20197 \pm 712 \\
.0\end{array}$ \\
\hline $\mathrm{X}_{2}(-)$ & \begin{tabular}{|c|}
$5.9 \pm 0.1$ \\
3 \\
\end{tabular} & $\begin{array}{c}3.5 \pm 0.1 \\
2 \\
\end{array}$ & $\begin{array}{c}46.9 \pm 1.5 \\
4\end{array}$ & $\begin{array}{c}20428 \pm 703 \\
.1\end{array}$ \\
\hline $\mathrm{R} 2(+)$ & \begin{tabular}{|c|}
$6.0 \pm 0.1$ \\
6 \\
\end{tabular} & $\begin{array}{c}3.6 \pm 0.1 \\
4\end{array}$ & $\begin{array}{c}48.4 \pm 1.7 \\
8 \\
\end{array}$ & $\begin{array}{c}21379 \pm 811 \\
.6\end{array}$ \\
\hline $\mathbf{R}_{2}(-)$ & \begin{tabular}{|c|}
$5.8 \pm 0.1$ \\
2 \\
\end{tabular} & $\begin{array}{c}3.3 \pm 0.1 \\
1\end{array}$ & $\begin{array}{c}44.8 \pm 1.3 \\
7\end{array}$ & $\begin{array}{c}19685 \pm 632 \\
.6\end{array}$ \\
\hline $\mathrm{W}(+)$ & \begin{tabular}{|c|}
$5.8 \pm 0.1$ \\
2 \\
\end{tabular} & $\begin{array}{c}3.4 \pm 0.1 \\
1 \\
\end{array}$ & $\begin{array}{c}45.2 \pm 1.4 \\
3 \\
\end{array}$ & $\begin{array}{c}19855 \pm 661 \\
.0\end{array}$ \\
\hline W $(-)$ & \begin{tabular}{|c|}
$6.0 \pm 0.1$ \\
5 \\
\end{tabular} & $\begin{array}{c}3.5 \pm 0.1 \\
4 \\
\end{array}$ & $\begin{array}{c}47.4 \pm 1.6 \\
9 \\
\end{array}$ & $\begin{array}{c}20933 \pm 763 \\
.8 \\
\end{array}$ \\
\hline$L^{\prime}(+)$ & \begin{tabular}{|c|}
$5.7 \pm 0.1$ \\
6 \\
\end{tabular} & $\begin{array}{c}3.3 \pm 0.1 \\
5\end{array}$ & $\begin{array}{c}43.8 \pm 1.9 \\
2 \\
\end{array}$ & $\begin{array}{c}19233 \pm 879 \\
.6\end{array}$ \\
\hline$L^{\prime}(-)$ & \begin{tabular}{|c|}
$6.0 \pm 0.1$ \\
1 \\
\end{tabular} & $\begin{array}{c}3.6 \pm 0.1 \\
1 \\
\end{array}$ & $\begin{array}{c}47.4 \pm 1.3 \\
1 \\
\end{array}$ & $\begin{array}{c}20928 \pm 602 \\
.0\end{array}$ \\
\hline $\mathrm{J}(+)$ & \begin{tabular}{|c|}
$5.9 \pm 0.1$ \\
4 \\
\end{tabular} & \begin{tabular}{|c|}
$3.5 \pm 0.1$ \\
3 \\
\end{tabular} & $\begin{array}{c}46.2 \pm 1.6 \\
9 \\
\end{array}$ & $\begin{array}{c}20180 \pm 774 \\
.8 \\
\end{array}$ \\
\hline $\mathbf{J}(-)$ & \begin{tabular}{|c|}
$5.9 \pm 0.1$ \\
2 \\
\end{tabular} & $\begin{array}{c}3.4 \pm 0.1 \\
1 \\
\end{array}$ & $\begin{array}{c}46.1 \pm 1.4 \\
3 \\
\end{array}$ & $\begin{array}{c}20413 \pm 654 \\
.7\end{array}$ \\
\hline $\mathrm{L}(+)$ & \begin{tabular}{|c|}
$5.8 \pm 0.1$ \\
3 \\
\end{tabular} & \begin{tabular}{|c|}
$3.4 \pm 0.1$ \\
2 \\
\end{tabular} & $\begin{array}{c}44.8 \pm 1.4 \\
4 \\
\end{array}$ & $\begin{array}{c}19494 \pm 652 \\
.6 \\
\end{array}$ \\
\hline $\mathrm{L}(-)$ & \begin{tabular}{|c|}
$6.0 \pm 0.1$ \\
4 \\
\end{tabular} & \begin{tabular}{|c|}
$3.6 \pm 0.1$ \\
3 \\
\end{tabular} & $\begin{array}{c}47.9 \pm 1.6 \\
6 \\
\end{array}$ & $\begin{array}{c}21463 \pm 772 \\
.2 \\
\end{array}$ \\
\hline$S_{2}(+)$ & \begin{tabular}{|c|}
$5.8 \pm 0.1$ \\
7 \\
\end{tabular} & $\begin{array}{c}3.4 \pm 0.1 \\
6 \\
\end{array}$ & $\begin{array}{c}45.5 \pm 1.9 \\
4 \\
\end{array}$ & $\begin{array}{c}19922 \pm 918 \\
.0\end{array}$ \\
\hline $\mathrm{S}_{2}(-)$ & \begin{tabular}{|c|}
$5.9 \pm 0.1$ \\
1 \\
\end{tabular} & $\begin{array}{c}3.5 \pm 0.1 \\
1 \\
\end{array}$ & $\begin{array}{c}46.4 \pm 1.3 \\
2 \\
\end{array}$ & $\begin{array}{c}20483 \pm 596 \\
.5 \\
\end{array}$ \\
\hline $\mathrm{H}^{\prime}(+)$ & \begin{tabular}{|c|}
$5.9 \pm 0.1$ \\
0 \\
\end{tabular} & $\begin{array}{c}3.5 \pm 0.1 \\
0\end{array}$ & $\begin{array}{c}46.5 \pm 1.1 \\
9\end{array}$ & $\begin{array}{c}20470 \pm 551 \\
.4\end{array}$ \\
\hline $\mathrm{H}^{\prime}(-)$ & \begin{tabular}{|c|}
$5.7 \pm 0.2$ \\
2 \\
\end{tabular} & $\begin{array}{c}3.3 \pm 0.2 \\
0\end{array}$ & $\begin{array}{c}44.8 \pm 2.5 \\
8 \\
\end{array}$ & $\begin{array}{c}19765 \pm 115 \\
5.9\end{array}$ \\
\hline (+) & \begin{tabular}{|c|}
$5.7 \pm 0.1$ \\
3 \\
\end{tabular} & $\begin{array}{c}3.3 \pm 0.1 \\
2 \\
\end{array}$ & $\begin{array}{c}44.4 \pm 1.4 \\
8 \\
\end{array}$ & $\begin{array}{c}19675 \pm 684 \\
.5 \\
\end{array}$ \\
\hline Z (-) & \begin{tabular}{|c|}
$6.0 \pm 0.1$ \\
4
\end{tabular} & $\begin{array}{c}3.6 \pm 0.1 \\
3\end{array}$ & $\begin{array}{c}48.1 \pm 1.6 \\
1 \\
\end{array}$ & $\begin{array}{c}21064 \pm 730 \\
.0\end{array}$ \\
\hline
\end{tabular}

\begin{tabular}{|c|c|c|c|}
\hline & months & & \\
\hline $\mathrm{A}_{1}(+)$ & $29.4 \pm 0.38$ & $148.9 \pm 8.33$ & $42.1 \pm 0.69$ \\
\hline $\mathrm{A}_{1}(-)$ & $29.8 \pm 0.28$ & $137.1 \pm 4.83$ & $42.4 \pm 0.44$ \\
\hline $\mathrm{A}_{2}(+)$ & $29.8 \pm 0.31$ & $138.5 \pm 5.96$ & $42.4 \pm 0.52$ \\
\hline $\mathrm{A}_{2}(-)$ & $29.5 \pm 0.32$ & $144.4 \pm 5.98$ & $42.3 \pm 0.54$ \\
\hline $\mathrm{G}_{3}(+)$ & $29.5 \pm 0.40$ & $127.7 \pm 5.97$ & $43.4 \pm 0.61^{*}$ \\
\hline $\mathrm{G}_{3}(-)$ & $29.8 \pm 0.27$ & $148.0 \pm 5.65^{*}$ & $41.8 \pm 0.47$ \\
\hline $\mathrm{O}_{1}(+)$ & $29.4 \pm 0.35$ & $125.2 \pm 7.08$ & $43.6 \pm 0.59^{*}$ \\
\hline $\mathrm{O}_{1}(-)$ & $29.8 \pm 0.29$ & $148.1 \pm 5.25^{*}$ & $41.8 \pm 0.47$ \\
\hline $\mathrm{Q}(+)$ & $29.3 \pm 0.34$ & $135.1 \pm 6.46$ & $43.1 \pm 0.56$ \\
\hline $\mathrm{Q}(-)$ & $30.0 \pm 0.29$ & $145.0 \pm 5.64$ & $41.8 \pm 0.50$ \\
\hline $\mathrm{Y}_{2}(+)$ & $29.7 \pm 0.40$ & $145.2 \pm 8.18$ & $42.1 \pm 0.74$ \\
\hline $\mathrm{Y}_{2}(-)$ & $29.7 \pm 0.27$ & $138.8 \pm 4.86$ & $42.5 \pm 0.42$ \\
\hline $\mathrm{A}_{2}(+)$ & $30.0 \pm 0.36$ & $139.2 \pm 6.12$ & $42.1 \pm 0.57$ \\
\hline$\overline{\mathrm{A}_{2}(-)}$ & $29.4 \pm 0.29$ & $142.4 \pm 5.85$ & $42.5 \pm 0.49$ \\
\hline $\mathrm{E}_{3}^{\prime}(+)$ & $29.4 \pm 0.37$ & $129.6 \pm 6.00$ & $43.4 \pm 0.60^{*}$ \\
\hline $\mathrm{E}_{3}{ }_{3}(-)$ & $29.8 \pm 0.28$ & $148.5 \pm 5.81^{*}$ & $41.7 \pm 0.47$ \\
\hline $\mathrm{C}_{1}(+)$ & $30.0 \pm 0.43$ & $150.8 \pm 8.50$ & $41.3 \pm 0.73$ \\
\hline $\mathrm{C}_{1}(-)$ & $29.5 \pm 0.26$ & $136.8 \pm 4.86$ & $42.8 \pm 0.43$ \\
\hline $\mathrm{C}_{2}(+)$ & $30.2 \pm 0.31^{*}$ & $144.0 \pm 6.09$ & $41.7 \pm 0.53$ \\
\hline $\mathrm{C}_{2}(-)$ & $29.2 \pm 0.32$ & $137.8 \pm 5.93$ & $43.1 \pm 0.52$ \\
\hline $\mathrm{E}(+)$ & $29.6 \pm 0.25$ & $140.2 \pm 4.73$ & $42.5 \pm 0.42$ \\
\hline E (-) & $30.2 \pm 0.50$ & $144.6 \pm 9.78$ & $41.5 \pm 0.83$ \\
\hline$X_{1}(+)$ & $28.8 \pm 0.40$ & $142.7 \pm 8.17$ & $43.1 \pm 0.72$ \\
\hline $\mathrm{X}_{1}(-)$ & $30.1 \pm 0.27^{* *}$ & $140.3 \pm 4.95$ & $41.9 \pm 0.43$ \\
\hline$X_{2}(+)$ & $29.7 \pm 0.34$ & $147.7 \pm 6.36$ & $41.9 \pm 0.56$ \\
\hline $\mathrm{X}_{2}(-)$ & $29.7 \pm 0.29$ & $134.3 \pm 5.63$ & $42.8 \pm 0.49$ \\
\hline $\mathrm{R}_{2}(+)$ & $29.4 \pm 0.39$ & $136.6 \pm 6.67$ & $42.9 \pm 0.58$ \\
\hline $\mathrm{R}_{2}(-)$ & $29.8 \pm 0.27$ & $143.6 \pm 5.51$ & $42.0 \pm 0.49$ \\
\hline $\mathrm{W}(+)$ & $29.8 \pm 0.30$ & $140.8 \pm 5.54$ & $42.2 \pm 0.51$ \\
\hline W (-) & $29.5 \pm 0.34$ & $141.4 \pm 6.66$ & $42.5 \pm 0.55$ \\
\hline $\mathrm{L}^{\prime}(+)$ & $30.2 \pm 0.37$ & $130.3 \pm 6.44$ & $42.5 \pm 0.56$ \\
\hline$L^{\prime}(-)$ & $29.4 \pm 0.28$ & $147.2 \pm 5.56^{*}$ & $42.2 \pm 0.50$ \\
\hline $\mathrm{J}(+)$ & $29.7 \pm 0.32$ & $138.8 \pm 6.37$ & $42.5 \pm 0.53$ \\
\hline $\mathbf{J}(-)$ & $29.7 \pm 0.31$ & $142.7 \pm 5.73$ & $42.2 \pm 0.52$ \\
\hline $\mathrm{L}(+)$ & $30.0 \pm 0.30$ & $134.1 \pm 5.39$ & $42.4 \pm 0.48$ \\
\hline $\mathrm{L}(-)$ & $29.2 \pm 0.34$ & $150.9 \pm 6.85$ & $42.2 \pm 0.59$ \\
\hline $\mathrm{S}_{2}(+)$ & $30.0 \pm 0.41$ & $139.9 \pm 7.93$ & $42.1 \pm 0.69$ \\
\hline $\mathrm{S}_{2}(-)$ & $29.5 \pm 0.27$ & $141.6 \pm 5.05$ & $42.4 \pm 0.45$ \\
\hline $\mathrm{H}^{\prime}(+)$ & $29.8 \pm 0.25$ & $139.7 \pm 4.76$ & $42.3 \pm 0.42$ \\
\hline $\mathrm{H}^{\prime}(-)$ & $29.4 \pm 0.49$ & $145.5 \pm 9.46$ & $42.3 \pm 0.80$ \\
\hline $\mathrm{Z}(+)$ & $29.6 \pm 0.31$ & $138.4 \pm 5.85$ & $42.6 \pm 0.53$ \\
\hline $\mathrm{Z}(-)$ & $29.8 \pm 0.33$ & $144.2 \pm 6.21$ & $42.0 \pm 0.53$ \\
\hline
\end{tabular}

\section{Discussion}

It was found that antigens $\mathrm{E}(80.6 \%), \mathrm{H}^{\prime}(77.5 \%)$, L (58.5\%), W (57.6\%), A 2 (57.0\%), Z (54.1\%), C (52.5\%) and $\mathrm{X}_{2}$ are most common in the herd under study. Antigens-markers of productive longevity are $\mathrm{O} 1$ antigen, of reproductive ability $-\mathrm{G}_{3}, \mathrm{O}_{1}$ and $\mathrm{L}^{\prime}$ antigens, of precocity $-\mathrm{X}_{1}$ antigen. The dependence of causes of exclusion of cows from the herd on the presence of certain antigens was not observed.

The results can be compared with the results obtained during similar studies on other breeds. I. Podrechneva isolated alleles-markers containing similar antigens from long-lived Kostroma cows [28]. Similar results were presented in the work by H. Valitov and S. Karamaev on 
Bestuzhev cows [29]. In other studies, O1 antigen was not mentioned as a marker of high productive longevity. On the contrary, in the work by E. Kondratyuk, this antigen was considered as one of the markers of low life expectancy [25].

Q antigen is isolated in Black-Motley and Aishir cows as the most frequently mentioned marker of high productive longevity $[25,30]$. For the same breeds, the main markers of low longevity are $\mathrm{O}^{\prime}, \mathrm{B}_{2}$, and $\mathrm{G}_{2}$ antigens $[25,30]$. There are works containing opposite results. In the work on Black-Motley cows by $\mathrm{M}$. Chasovschikova, $\mathrm{B}_{2}$ antigen is associated with a high life expectancy, and Q antigen is associated with low lifetime productivity [24]. Similar results were obtained for Bestuzhev cows in the work by $\mathrm{H}$. Valitov and $\mathrm{S}$. Karamaev [29].

\section{Conclusion}

Thus, each breed has a unique set of antigens-markers of productive longevity and reproductive ability, which indicates the relevance of research on the Red-Motley breed.

This work was financially supported by the Ministry of Science and Higher Education of Russia, state registration number R\&D: AAAA-A19-119012290066-7.

\section{References}

1. O.V. Ivanova, E.A. Ivanov, V.A. Tereschenko, L.V. Efimova, T.V. Zaznobina, O.A. Frolova, IOP Conference Series: Earth and Environmental Science, 341, 8 (2019). doi: 10.1088/17551315/341/1/012095

2. V. Bekenev, Agricultural biology, 54(4), 655-666 (2019)

3. T. Pritchard, M. Coffey, R. Mrode, E. Wall, Animal., $\begin{array}{llll}7(1) & 34-46 \quad \text { (2013) }\end{array}$ $10.1017 / \mathrm{S} 1751731112001401$

4. M.C. Lucy, J. Dairy Sci., 102, 3706-3721 (2019) https://doi.org/10.3168/jds.2018-15544

5. V. Deeva, Siberian Bulletin of Agricultural Science, 2(206), 35-39 (2010)

6. R. Milostivy, L. Karlova, Breeding and genetics of animals, 54, 65-74 (2017)

7. G. Serdyuk, Dairy and beef cattle breeding, 6, 7-10 (2015)

8. E. Bydantseva, O. Kavardakova, News of Orenburg State Agrarian University, 5(37), 114-116 (2012)

9. V.I Shlyakhtunov, E.M. Karpovich, Actual problems of the intensive development of animal husbandry, 13(2) (2010) Retrieved from: https://cyberleninka.ru/article/ n/dolgoletie-ipozhiznennaya-molochnaya-produktivnostdocherey-raznyh-bykov-proizvoditeley

10. V.G. Kahikalo, S.V. Naumov, Bulletin of KrasSAU, 10, 94-97 (2009)
11. M.A. Pilipenko, Agrarian Bulletin of the Urals, 12-2 (92), 46-48 (2011)

12. O.R. Kurchenkova, M.Yu. Petrova, Yu.V. Chernikov, Bulletin of Omsk State Agrarian University, 4, 42-47 (2017)

13. J. Ogorevc, T. Kunej, A. Razpet, P. Dovc, Animal Genetics, 40, 832-851 (2009) doi:10.1111/j.13652052.2009.01921.x

14. N. Yudin, M. Voevoda, Genetics, 51(5), 600-612 (2015). doi: 10.7868/S0016675815050082

15. Q. Zhang, B. Guldbrandtsen, J. Thomasen, M. Lund, G. Sahana. Journal of Dairy Science, 99(9), 72897298 (2016). doi: 10.3168/jds.2015-10697

16. N. Yudin, L. Vasilyeva, V. Belyavskaya, R. Aitnazarov, P. Smirnov, M. Heaton, U. Legreid, G. Orlova, A. Romashchenko, M. Voevoda, Vavilov Journal of Genetics and Breeding, 18(3), 463-468 (2014)

17. G.P. Kovaleva, M.N. Lapina, N.V. Suliga, V.A. Vitol, Collection of scientific papers of the AllRussian Research Institute of Sheep and Goat Breeding, 2(10), 54-62 (2017)

18. O. Shatalina, Agricultural biology, 53(2), 309-317 (2018). doi: 10.15389/agrobiology.2018.2.309rus

19. S. Tyulebaev, M. Kadysheva, L. Surundaeva, P. Tikhonov, News of Orenburg State Agrarian University, 4(32), 321-322 (2011)

20. E. Shukurova, Far Eastern Agricultural Bulletin, 2(42), 111-119 (2017)

21. L. Kholodova, K. Novoselova. The use of immunogenetics in the selection of the dairy herd of the Republic of Mari El, Bulletin of Mari State University, 4(3), 69-76 (2018). DOI: 10.30914/2411-9687-2018-4-3-69-76

22. M. Selionova, G. Kovalev, M. Lapin, N. Sulyga, V. Vitol, Dairy Newsletter, 2(26), 53-59 (2017)

23. A. Konovalov, A. Ilyin, M. Abramov, N. Kosyachenko, T. Grigoriev, AgroZooEngineering, 2(2) (2018). DOI: 10.15838/alt.2018.2.2.3

24. M. Chasovshchikova, News of Orenburg State Agrarian University, 6(44), 81-83 (2013)

25. E. Kondratyuk, Bulletin of the Russian Agricultural Science, 5, 69-72 (2019)

26. N. Kulumaev, G. Goncharenko, Siberian Bulletin of Agricultural Science, 10, 59-64 (2007)

27. S.L. Gridina, O.V. Shatalina, Achievements of science and technology of agribusiness, 6, 69-70 (2011)

28. I. Podrechnev, Veterinary medicine, animal husbandry, biotechnology, 3, 54-59 (2019)

29. H. Valitov, S. Karamaev, Bulletin of the Lower Volga Agricultural University: science and higher professional education, 3(23), 98-102 (2011)

30. M. Boev, E. Kukushka, M. Boev, Problems of biology of productive animals, 4, 23-25 (2011) 